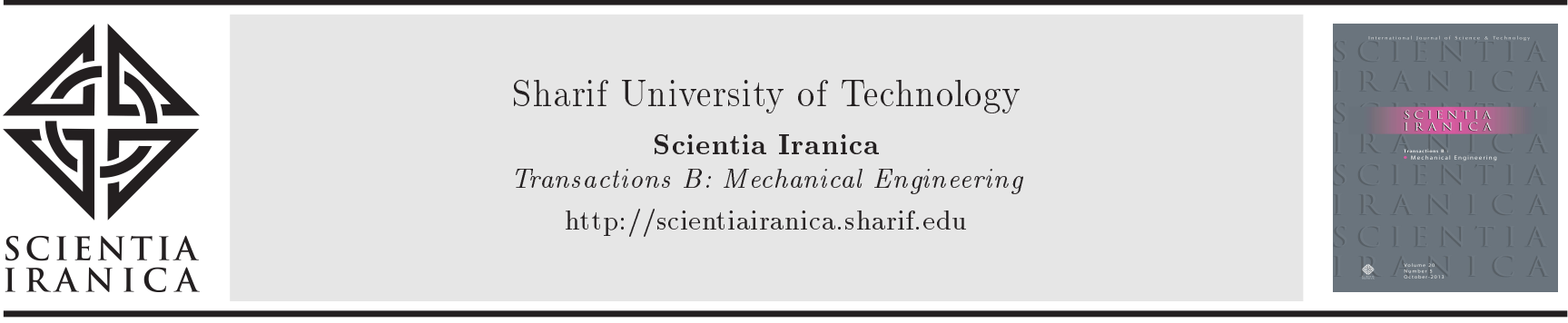

\title{
Numerical 3D simulation of developing turbulent stratified gas-liquid flow in curved pipes consisting of entrained particles through this type of flow
}

\author{
M.A. Rasoulian ${ }^{\mathrm{a}}$, A. Rasteh ${ }^{\mathrm{a}}$, A. Farokhipour ${ }^{\mathrm{a}}$, Z. Mansoori ${ }^{\mathrm{b}}$, \\ M. Saffar-Avval ${ }^{\mathrm{a}, *}$, A. Haj ${ }^{\mathrm{b}}$, and G. Ahmadi ${ }^{\mathrm{c}}$ \\ a. Department of Mechanical Engineering, Amirkabir University of Technology, Tehran, Iran. \\ b. Energy Research Center, Amirkabir University of Technology, Tehran, Iran. \\ c. Department of Mechanical and Aeronautical Engineering, Clarkson University, Potsdam, NY, USA. \\ Received 6 May 2018; accepted 27 August 2018
}

\section{KEYWORDS}

Gas-liquid multiphase

flow;

Stratified flow;

Particle tracking;

Curved pipe;

Numerical analysis.

\begin{abstract}
Since curved pipes are widely used in industrial equipment, predicting multiphase flows in these geometries is of great importance. In the present study, a computational model for predicting the velocity profile is developed and used to study the developing turbulent gas-liquid flows in curved pipes. In order to discretize and solve the three-dimensional steady-state momentum equations, the finite volume scheme on staggered grids as well as central difference and QUICK scheme have been used. Moreover, the $k-\varepsilon$ model has been employed to reflect the nature of turbulence in the flow. In order to address the needs for faster convergence and convenient mapping of the physical domain, the computations have been performed in a newly extended coordinate system. Particle tracking has been done using Lagrangian approach in which a two-way coupling regime has been considered. In terms of validation, the numerical simulation results for the straight pipe have been compared with the analytical solution and previous experimental results. Moreover, injection of particles through the flow indicates that, in each section of the bend, the trade-off between centrifugal and pressure gradient forces plays a key role in particles' motion. In the last section, the effects of particle diameter and bend curvature on particles' motion have been examined.
\end{abstract}

(C) 2018 Sharif University of Technology. All rights reserved.

\section{Introduction}

Behavior of multi-phase flows in curved pipes is of use for many industrial applications. Since bends are widely exploited, particularly in pieces of equipment

\footnotetext{
*. Corresponding author.

E-mail addresses: ali.rasoulian@aut.ac.ir (M.A. Rasoulian); aminrasteh@aut.ac.ir (A. Rasteh); farokhipour_ali@aut.ac.ir (A. Farokhipour); z.mansoori@aut.ac.ir (Z. Mansoori); Mavval@aut.ac.ir (M. Saffar-Avval); alihaj@aut.ac.ir (A. Haj);gahmadi@clarkson.edu (G.Ahmadi)
}

of gas and oil industry, the computational modeling of such flows in these geometries can help predict the flow behavior passing through them to a large extent. The stratified flow velocity profiles of both gas and liquid phases, pressure losses along the passage, and secondary flows occurring along the pipe are significantly affected by the curved section. Furthermore, in the production procedures, since the entrainment of sand particles to transmission lines frequently carrying gasliquid flows is an inevitable issue during this process, their behavior should be carefully considered to prevent probable consequences such as pressure loss, erosion, and equipment failure. Among the geometries, bends 
are more prone to erosion phenomenon due to the impingement of particles on the wall downstream of the curved section at which particles, because of their inertia, are not able to follow the streamlines and, consequently, detach from them and impact the inner surface of the pipe. Many pieces of research available in the literature, mostly conducted experimentally, are devoted mainly to the pressure drop across the bend, bulk flow measurements, and mean void fraction of gas and liquid phases $[1,2]$. In many applications, however, more detailed information of turbulent stratified flow, such as gas and liquid velocity profiles, as well as the location and strength of secondary flows, can facilitate the prediction of the particle's motion and erosion wear caused by entrained particles in multiphase flows through the bend.

The multi-phase flows in curved geometries are generally more complex than those in straight ones are. This may be the reason why there is a rather limited amount of research in this area. The available literature includes semi-empirical studies; for instance, Gardner and Neller [3] implemented visual and experimental studies on bubble/slug flows using a pipe of $76 \mathrm{~mm}$ diameter in a vertical $90^{\circ}$ elbow with radii of curvatures of 305 and $610 \mathrm{~mm}$. In their work, they used a traversing probe to measure the local time-averaged air concentration. They reported that gas could flow either on the outside or the inside of the bend depending on the balance between the centrifugal force and the gravity. The trade-off between these two forces is expressed in terms of Froude number:

$$
F r_{\theta}=V^{2} / g a \sin \theta
$$

where $V$ is the mixture mean velocity, $a$ is the radius of curvature of the bend, and $\theta$ is the stream-wise angle across the bend. It is noteworthy that the phases are flowing in radial equilibrium when the Froude number is equal to unity. For $F r>1$, the gas is displaced towards the inside of the bend, while, for $F r<1$, the gas moves to the outside of the bend. An experimental study was performed on two-phase mixture flowing in an inverted U-bend by Usui et al. [4,5]. They measured some figures such as the local void fraction distribution, averaged void fraction over the bend with the aid of quick closing valves, and slip ratio both through and in the vicinity of the bend. As a result of their work, they found that the secondary flow in bend would have governing effects upon the distribution of void fraction across the whole crosssection of the pipe. Das et al. [6] and Bandyopadhyay et al. [7] performed an experimental study of gas/nonNewtonian liquid flow through a $12.7 \mathrm{~mm}$ curved pipe. They managed to develop an empirical correlation to estimate the pressure drop over the passage as a function of dynamic variables and physical parameters of the system. In another investigation by Ribeiro et al. [8], drop sizes upstream and downstream of a $90^{\circ}$, $32 \mathrm{~mm}$ internal diameter bend on a horizontal plane were measured. Their results demonstrated that the drop sizes downstream were $50-75 \%$ of those upstream. From their point of view, this trend was attributed to thicker films caused by the initial deposition induced by the secondary flow that occurred in the gas phase. Moreover, they found that the ratio of downstream to upstream entrained fraction would decrease with a rise in the upstream entrainment fraction. Azzi et al. [9] considered different correlations to estimate the pressure loss of the variety of flow conditions, the bend orientation, and geometries; eventually, they decided that in order to calculate adequately the two-phase flow pressure loss in both vertical and horizontal ducts in wider parameter ranges, the effect of gravitational force in experiments should be taken into account.

Comprehensive studies concerning the effect of the bend on the flow fields of a two-phase air/water mixture were performed by Wang et al. in horizontal $[10,11]$ and vertical return bends $[12,13]$. In these studies, for the horizontal experiments, return bends with $3-6.9 \mathrm{~mm}$ diameter and curvature ratios (curvature radius/pipe diameter) of $1.5,2.5$, and 3.5 were used, while a single bend geometry $(6.9 \mathrm{~mm}$ ID and curvature ratio of 1.5 ) was exploited for the vertical cases, and the distribution of the phases was observed through still photography. In their investigation, the effects of parameters, such as tube size, mass flux, and curvature ratio, were examined. Finally, with the aid of visual observation in the horizontal bends, the transition of flow regime from stratified to annular flows was detected. On the other hand, for the vertical return bend with $D=6.9 \mathrm{~mm}$, some unique phenomena, such as flow reversal and frozen slug flow, were observed absent in previously reported results for horizontal configurations. However, such phenomena (the flow reversal and freezing slug) were hardly seen at a smaller tube ( $D=3 \mathrm{~mm}$ ) owing to the effect of the surface tension and flow inertia.

Spedding and Benard [14] measured the pressure drop of a two-phase air/water pipe flow and threephase air/oil/water through a $26 \mathrm{~mm}$ ID pipe and elbow (curvature radius/pipe diameter $=0.654$ ) for vertical to horizontal flows. Two-phase results were highly dependent on the flow regimes present in the system. Three-phase data also showed noticeable, yet undramatic, differences. Recently, several investigators have implemented a few local measurements across an elbow by a wire-mesh sensor. Abdulkadir et al. [15] considered the flow fields of a $90^{\circ}$ bend (curvature radius/pipe diameter $=2.3$ ) with the aid of advanced instrumentation, including Wire-Mesh Sensor (WMS) and Electrical Capacitance Tomography (ECT), to produce cross-sectionally resolved data on void fraction 
and high-speed video. Although, for the vertical $90^{\circ}$ bend, they observed that bubble, stratified, slug, and semi-annular flows were present downstream, the flow regime showed the same configurations upstream of the bend for the horizontal $90^{\circ}$ bend. As a result of another work of theirs, they found that ECT and WMS predicted the same flow pattern signatures. Furthermore, in both the vertical and horizontal $90^{\circ}$ bends, the gravitational force tends to move the liquid and gas to the inside and outside of the bend, respectively. Different slug characteristics, such as the liquid slug body length distribution, were identified by Kesana [16]. They also obtained the frequency of the slugs from void fraction data using dual wiremesh sensors in the horizontal flow orientation. In their investigation, they examined the discrepancies between the properties of the slug flow and pseudo-slug flow regimes upstream and downstream of an elbow. In another study involving a dual wire-mesh sensor, Vieira et al. [17] focused on the two-phase stratified-slug transition, stratified wavy, and annular flow regimes before and after a horizontal stainless steel elbow with an internal diameter of $76.2 \mathrm{~mm}$ and $144 \mathrm{~mm}$ radii of curvature. Time series analysis of void fraction data from WMS resulted in determining mean void fraction, local time-averaged void fraction distribution, liquid phase distribution around the peripheral surface, interfacial structure velocities, as well as characteristic signatures within the cross-section of pipe before and after the elbow. In their research, the effect of some factors, such as the superficial liquid and gas velocities and liquid viscosity, on the measured parameters were examined. The results indicated that the distribution of gas and liquid phases and interfacial velocities were significantly changed, even for $20 D$ downstream of the elbow. A comprehensive experimental study of gassolid heat transfer in a pipe with different configurations (horizontal, inclined, and vertical positions) was implemented by Mokhtarifar et al. [18]. The effects of parameters, such as pipe slope and feed rate of solid particles, on the Nusselt number were examined. Results showed that the Nusselt number, in a dilute regime of the mixture, decreased to a lower feed rate of solids; conversely, at higher feed rates of solids, it increased. Importantly, Nusselt number took higher values close to the pipe position of $45^{\circ}$.

In terms of CFD simulations, Nojabaei et al. [19] investigated heat transfer in a turbulent liquid-solid suspension upward flow in a vertical pipe where a four-way coupling regime was considered. During the simulation, the effects of loading ratio, particle density, and particle diameter on heat transfer coefficient were discussed. The results illustrated that particle to liquid density had a prominent effect on heat transfer and turbulence characteristics. The turbulent heat transfer in gas-solid flows through an inclined pipe with various inclination angles under a constant wall heat flux condition was investigated by Pishvar et al. [20]. According to the report of their study, an increase up to a certain angle led to a significant surge in Nusselt number. Dabirian et al. [21] conducted a research study using a commercial CFD code for 4-in horizontal pipe carrying stratified turbulent air/water and presented the results such as liquid holdup, liquid height, wall shear stress, and velocity profiles. Haj et al. [22] used a new form of the toroidal coordinate system to address the need for convenient mapping of the physical domain, where fluid flow pattern was considered laminar and stratified. In addition, they employed bipolar coordinate in curved pipe crosssection. They achieved results by assuming the effect of liquid phase as a boundary condition at interfacial surface and considering the angular displacement of liquid phase negligible. They reported that several vortices were formed in the gas phase whose size and strength varied along the curved section.

Currently, simultaneous Particle Image Velocimetry (PIV) measurements of stratified turbulent air/water flow in a horizontal pipe were performed using water droplets as tracers in the gas phase by Ayati et al. [23]. The two-phase flow measurements indicated the expected behavior of low flow rates and very interesting features related to the turbulence structure at higher flow rates that led to wavy interfaces. Inspired by the ongoing approach, Vest $\phi$ l et al. [24] produced detailed velocity profile measurements over a range of operating conditions of a two-phase gas/liquid flow with low liquid fractions in horizontal and inclined pipes. The experiments were implemented in a $15 \mathrm{~m}$ long stainless steel pipe with internal diameter of $56 \mathrm{~mm}$ at room temperature and atmospheric outlet pressure. In general, higher axial velocities were observed in the gas phase. Higher velocity gradients were observed close to the pipe wall and interface. Based on the horizontal gas-liquid flow measurements, the maximum velocity was located close to halfway between the interface and the upper wall.

Since the entrainment of solid particles into pipes carrying crude oil is an inevitable and inseparable part of production procedure in oil and gas industry, prediction of particles behaviour, particularly particle deposition, can prevent operational problems such as a decrease in production, excessive pressure drop, erosion, corrosion, and, consequently, equipment failure. The operational condition, in which continuous movement of particles through the pipe is guaranteed, will be the best approach to confront the deposition issue. This condition can be managed by operating above the critical particle deposition velocity, which is the velocity that satisfies the desired operational condition. In order to address this requirement, a few investigations regarding the particles in two-phase 
flows were performed. Stevenson and Thorpe [25] experimentally measured the average velocity of particles in a smooth stratified flow through curved pipes. They found that particle velocity followed a linear trend with respect to the average velocity of the liquid phase of the flow. Moreover, it was seen that larger particles moved more quickly than smaller ones along the pipe. As a result of their experiments, they found that particle velocity decreased with increasing the liquid viscosity. This could be justified by considering the particle size relative to the depth of the viscous sublayer. Another experimental study was conducted by Dabirian et al. [26] in a 4-in horizontal pipe carrying a stratified flow to examine the behaviour of solid particles (glass beads) with concentration ranges of 100 to $10,000 \mathrm{ppm}$. In their research, flow regime sequences of three ranges of particle sizes were investigated. They introduced gas velocity, particle concentration, and liquid holdup as key parameters for the creation of different particle regime flows. Furthermore, they reported that the dependence of critical particle deposition velocity on particle size increased as their sizes increased; by the same token, the critical particle deposition velocity rose. In addition, when the concentration of particles increased, the critical velocity remained fairly unchanged for the smaller particles, while it gradually increased for larger ones $(\geq 125 \mu \mathrm{m})$. Moreover, some of the most important characteristics of the stratified air-water flow in a mini-channel were studied by means of a rule-based fuzzy inference system by ZehtabiyanRezaie [27]. Their analysis demonstrated that the system was a viable approach to avoid significant computation cost of the numerical simulations. In the case of CFD prediction of erosion phenomenon, Banakermani et al. [28] simulated a range of elbow angles for two different flow orientations; horizontal inlet and outlet flow directions ( $\mathrm{H}-\mathrm{H}$ flow) and the cases with vertical inlet and outlet flows on the horizontal plane, or with some angles (V-H flow). They found that the total annual eroded volume in the $\mathrm{H}-\mathrm{H}$ case configuration was larger than that in $\mathrm{V}-\mathrm{H}$ case figure. Moreover, their results showed that, in both $\mathrm{V}-\mathrm{H}$ and
$\mathrm{H}-\mathrm{H}$ cases, over the range of mass loadings under investigation, the maximum erosion rate increased gradually when the elbow angle increased from $15^{\circ}$ to $90^{\circ}$; however, the rate of total eroded volume remained relatively constant over the sand rate range.

The thrust of this work is to study the developed turbulent stratified gas-liquid in horizontal curved pipes consisting of entrained particles. A computational model of predicting velocity profiles and secondary flows has been developed. The threedimensional steady-state momentum equations, by means of the $k-\varepsilon$ turbulence model, have been discretized and solved using the finite volume scheme. The computations have been performed in the extended form of toroidal and bipolar coordinate systems. Axial velocity contours and secondary flows have been predicted in the gas phase in the curved pipe in four axial sections over the bend. Particle tracking has been implemented by the Lagrangian approach considering the two-way coupling regime. The behavior of particles has been discussed in three cross-sectional positions of the pipe along the curved section, and the effects of particle diameter and bend curvature on particles' motion have been examined. Experimental results and analytical data for the case of the developed stratified gas-liquid flow in curved pipes have been used to validate obtained results.

\section{Mathematical formulation}

\subsection{Coordinate system}

In this study, in order to match the coordinate system with the flow configuration in curved geometries, the orthogonal toroidal coordinate system is used to simulate the turbulent stratified flow in curved pipes. The toroidal coordinate system is depicted in Figure 1. The liquid and gas domains in stratified pipe flow, as shown in Figure 1, are modelled by the bipolar coordinate system in the pipe cross-section described as follows:

$$
x=c \frac{\sinh \eta}{\cosh \eta-\cos \xi},
$$

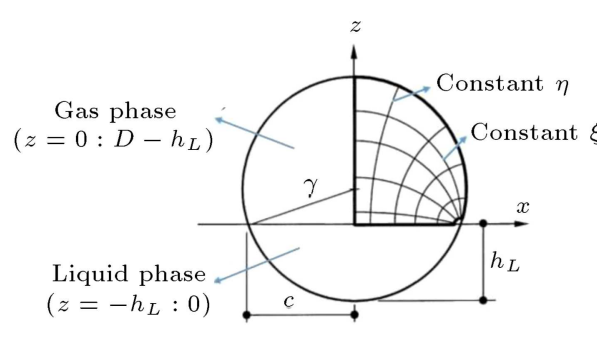

(a)

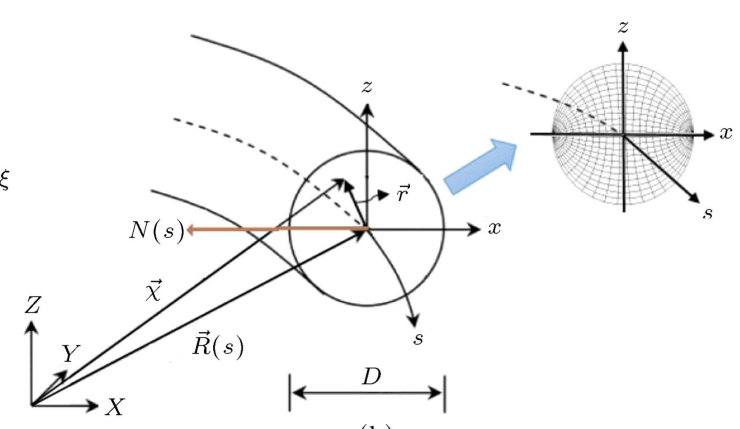

(b)

Figure 1. (a) Schematic diagram of the new toroidal coordinates system $(\xi, \eta, s)$. (b) The bipolar coordinate at the pipe cross-section. 


$$
\begin{aligned}
& z=c \frac{\sinh \eta}{\cosh \eta-\cos \xi}, \\
& \begin{cases}\gamma \leq \xi<\pi, & -\infty<\eta<\infty \\
\pi \leq \xi<\pi+\gamma, & \text { for upper fluid( air) }\end{cases} \\
& \quad \text { for lower fluid (water) }
\end{aligned}
$$

where $c$ and $\gamma$, as illustrated in Figure 1, are focal length in bipolar coordinate system and the half-view angle of the interface from the centre of the pipe, respectively. In other words, $\gamma$ is equal to half of the angle subtended by the centre of the pipe and the gasliquid interface and is defined as follows:

$$
\gamma=\cos ^{-1}\left[1-\frac{2 h_{L}}{D}\right] \text {. }
$$

Herein, $h_{L}$ and $D$ represent the liquid height and pipe internal diameter, respectively. In this study, in order to address the needs for faster convergence and convenient mapping of the physical domain, a new form of the toroidal coordinate system is created and used. Therefore, the Navier-Stokes governing equations need to be stated in the frame of the aforementioned coordinate system. Accordingly, a bipolar coordinate system should be expressed in the reference Cartesian coordinate system $X Y Z$. The position of any point through the curved geometry can be defined by vector $\vec{\chi}$. Thus, $\vec{\chi}$ can be calculated by:

$$
\vec{\chi}=\vec{R}(s)+\vec{r},
$$

where $\vec{R}(s)$ and $\vec{r}$ (in the reference Cartesian coordinate system $X Y Z)$ are described as follows:

$$
\begin{aligned}
& \vec{R}(s)=a \cos \left(\frac{s}{a}\right) \widehat{i}+a \sin \left(\frac{s}{a}\right) \widehat{j}, \\
& \vec{r}=c \frac{\sinh \eta}{\cosh \eta-\cos \xi} \vec{N}(s)+c \frac{\sinh \eta}{\cosh \eta-\cos \xi} \widehat{k},
\end{aligned}
$$

where $a$ is the radius of curvature of the curve, $s$ is axial direction (stream-wise direction), and $\vec{N}(s)$ represents the principal normal vector of $s$ and is described by:

$$
\vec{N}(s)=-\cos \left(\frac{s}{a}\right) \hat{i}-\sin \left(\frac{s}{a}\right) \hat{j}
$$

Thus, the position of each point inside the curve in the reference Cartesian coordinate system $X Y Z$ can be defined by vector $\vec{\chi}$ as follows:

$$
\begin{aligned}
\vec{\chi}= & {\left[a \cos \left(\frac{s}{a}\right)+c \frac{\sinh \eta}{\cosh \eta-\cos \xi} \cos \left(\frac{s}{a}\right)\right] \widehat{i} } \\
& +\left[a \sin \left(\frac{s}{a}\right)+c \frac{\sinh \eta}{\cosh \eta-\cos \xi} \sin \left(\frac{s}{a}\right)\right] \widehat{j} \\
& +c \frac{\sin \eta}{\cosh \eta-\cos \xi} \widehat{k} .
\end{aligned}
$$

The corresponding scale factors (metric coefficients) are provided by:

$$
\begin{aligned}
h_{\xi} & =\sqrt{\left(\partial \chi_{X} / \partial \xi\right)^{2}+\left(\partial \chi_{Y} / \partial \xi\right)^{2}+\left(\partial \chi_{Z} / \partial \xi\right)^{2}} \\
& =\frac{1}{\cosh \eta \cos \xi}, \\
h_{\eta} & =\sqrt{\left(\partial \chi_{X} / \partial \eta\right)^{2}+\left(\partial \chi_{Y} / \partial \eta\right)^{2}+\left(\partial \chi_{Z} / \partial \eta\right)^{2}} \\
& =\frac{1}{\cosh \eta-\cos \xi}, \\
h_{s} & =\sqrt{\left(\partial \chi_{X} / \partial s\right)^{2}+\left(\partial \chi_{Y} / \partial s\right)^{2}+\left(\partial \chi_{Z} / \partial s\right)^{2}} \\
& =1+\frac{c \sinh \eta}{a(\cosh \eta-\cos \xi)} .
\end{aligned}
$$

\subsection{Governing equations}

The Reynolds-Averaged Navier-Stokes (RANS) continuity and momentum equations for steady-state incompressible and viscous flow in the curvilinear new coordinates system $(\xi, \eta, s)$ are given as follows [29]:

- Continuity:

$$
\frac{1}{h_{\xi} h_{\eta} h_{s}}\left[\frac{\partial\left(h_{\eta} h_{s} u\right)}{\partial \xi}+\frac{\partial\left(h_{\xi} h_{s} v\right)}{\partial \eta}+\frac{\partial\left(h_{\xi} h_{\eta} w\right)}{\partial s}\right]=0
$$

- $\xi$ momentum:

$$
\begin{aligned}
\frac{1}{h_{\xi} h_{\eta} h_{s}}[ & \frac{\partial\left(\rho h_{\eta} h_{s} U U\right)}{\partial \xi}+\frac{\partial\left(\rho h_{\xi} h_{s} V U\right)}{\partial \eta} \\
& \left.+\frac{\partial\left(\rho h_{\xi} h_{\eta} W U\right)}{\partial s}\right] \\
& +\frac{\rho V}{h_{\xi} h_{\eta}}\left(U \frac{\partial h_{\xi}}{\partial \eta}-V \frac{\partial h_{\eta}}{\partial \xi}\right) \\
& -\frac{\rho W}{h_{\xi} h_{s}}\left(W \frac{\partial h_{s}}{\partial \xi}\right) \\
& =-\frac{1}{h_{\xi}} \frac{\partial p}{\partial \xi}+\frac{\mu}{h_{\xi}} \frac{\partial}{\partial \xi}\left\{\frac { 1 } { h _ { \xi } h _ { \eta } h _ { s } } \left[\frac{\partial\left(\rho h_{\eta} h_{s} U U\right)}{\partial \xi}\right.\right. \\
& \left.\left.+\frac{\partial\left(\rho h_{\xi} h_{s} V U\right)}{\partial \eta}+\frac{\partial\left(\rho h_{\xi} h_{\eta} W U\right)}{\partial s}\right]\right\} \\
& -\frac{\mu}{h_{\eta} h_{s}}\left[\frac { \partial } { \partial \eta } \left(\frac { h _ { s } } { h _ { \xi } h _ { \eta } } \left(\frac{\partial\left(h_{\eta} V\right)}{\partial \xi}\right.\right.\right. \\
& \left.\left.-\frac{\partial\left(h_{\xi} V\right)}{\partial \eta}\right)\right)-\frac{\partial}{\partial s}\left(\frac { h _ { \eta } } { h _ { \xi } h _ { s } } \left(\frac{\partial\left(h_{\xi} V\right)}{\partial s}\right.\right.
\end{aligned}
$$




$$
\begin{aligned}
& \left.\left.\left.-\frac{\partial\left(h_{s} V\right)}{\partial \xi}\right)\right)\right]+\rho g_{\xi}+S_{p}^{u} \\
& +\nabla \cdot\left(-\overrightarrow{\rho u^{\prime} \overrightarrow{v^{\prime}}}\right) .
\end{aligned}
$$

- $\eta$ momentum:

$$
\begin{aligned}
& \frac{1}{h_{\xi} h_{\eta} h_{s}}\left[\frac{\partial\left(\rho h_{\eta} h_{s} U V\right)}{\partial \xi}+\frac{\partial\left(\rho h_{\xi} h_{s} V V\right)}{\partial \eta}\right. \\
& \left.+\frac{\partial\left(\rho h_{\xi} h_{\eta} W V\right)}{\partial s}\right] \\
& +\frac{\rho U}{h_{\xi} h_{\eta}}\left(V \frac{\partial h_{\eta}}{\partial \xi}-U \frac{\partial h_{\xi}}{\partial \eta}\right) \\
& -\frac{\rho W}{h_{s} h_{\eta}}\left(W \frac{\partial h_{s}}{\partial \eta}\right) \\
& =-\frac{1}{h_{\eta}} \frac{\partial p}{\partial \eta}+\frac{\mu}{h_{\eta}} \frac{\partial}{\partial \eta}\left\{\frac { 1 } { h _ { \xi } h _ { \eta } h _ { s } } \left[\frac{\partial\left(\rho h_{\eta} h_{s} U V\right)}{\partial \xi}\right.\right. \\
& \left.\left.+\frac{\partial\left(\rho h_{\xi} h_{s} V V\right)}{\partial \eta}+\frac{\partial\left(\rho h_{\xi} h_{\eta} W V\right)}{\partial s}\right]\right\} \\
& -\frac{\mu}{h_{\xi} h_{s}}\left[\frac { \partial } { \partial s } \left(\frac { h _ { \xi } } { h _ { \eta } h _ { s } } \left(\frac{\partial\left(h_{s} W\right)}{\partial \eta}\right.\right.\right. \\
& \left.\left.-\frac{\partial\left(h_{\eta} V\right)}{\partial s}\right)\right)-\frac{\partial}{\partial \xi}\left(\frac { h _ { s } } { h _ { \xi } h _ { \eta } } \left(\frac{\partial\left(h_{\eta} V\right)}{\partial \xi}\right.\right. \\
& \left.\left.\left.-\frac{\partial\left(h_{\xi} V\right)}{\partial \eta}\right)\right)\right]+\rho g_{\eta}+S_{p}^{v} \\
& +\nabla \cdot\left(-\overline{\rho v^{\prime} \overrightarrow{v^{\prime}}}\right)
\end{aligned}
$$

- $s$ momentum:

$$
\begin{aligned}
\frac{1}{h_{\xi} h_{\eta} h_{s}}\left[\frac{\partial\left(\rho h_{\eta} h_{s} U W\right)}{\partial \xi}+\frac{\partial\left(\rho h_{\xi} h_{s} V W\right)}{\partial \eta}\right. \\
\left.+\frac{\partial\left(\rho h_{\xi} h_{\eta} W W\right)}{\partial s}\right]+\frac{\rho U}{h_{\xi} h_{s}}\left(W \frac{\partial h_{s}}{\partial \xi}\right) \\
+\frac{\rho V}{h_{\eta} h_{s}}\left(W \frac{\partial h_{s}}{\partial \eta}\right) \\
=-\frac{1}{h_{s}} \frac{\partial p}{\partial s}+\frac{\mu}{h_{s}} \frac{\partial}{\partial s}\left\{\frac { 1 } { h _ { \xi } h _ { \eta } h _ { s } } \left[\frac{\partial\left(\rho h_{\eta} h_{s} U W\right)}{\partial \xi}\right.\right. \\
\left.\left.+\frac{\partial\left(\rho h_{\xi} h_{s} V W\right)}{\partial \eta}+\frac{\partial\left(\rho h_{\xi} h_{\eta} W W\right)}{\partial s}\right]\right\} \\
\\
-\frac{\mu}{h_{\xi} h_{\eta}}\left[\frac { \partial } { \partial \xi } \left(\frac { h _ { \eta } } { h _ { \xi } h _ { s } } \left(\frac{\partial\left(h_{\xi} U\right)}{\partial s}\right.\right.\right.
\end{aligned}
$$

$$
\begin{aligned}
& \left.\left.-\frac{\partial\left(h_{s} W\right)}{\partial \xi}\right)\right)-\frac{\partial}{\partial s}\left(\frac { h _ { \xi } } { h _ { \eta } h _ { s } } \left(\frac{\partial\left(h_{s} W\right)}{\partial \eta}\right.\right. \\
& \left.\left.\left.-\frac{\partial\left(h_{\eta} V\right)}{\partial s}\right)\right)\right]+\rho g_{s}+S_{p}^{w} \\
& +\nabla \cdot\left(-\overline{\rho w^{\prime} \overrightarrow{v^{\prime}}}\right)
\end{aligned}
$$

Herein, $u, v, w$ are the velocity components, $U, V, W$ are the mean velocity components, $u^{\prime}, v^{\prime}, w^{\prime}$ are the fluctuation velocity components, and $S_{p}^{u}, S_{p}^{v}, S_{p}^{w}$ are the sources or sink terms when the particles exist in the flow field, and the career fluid is influenced by them (two-way coupling regime); in $\xi, \eta$, and $s$ directions, they are respectively defined by:

$$
\begin{aligned}
& S_{p}^{u}=\varphi_{p}\left\langle-\rho_{p}\left[\frac{d u_{p}}{d t}-g_{\xi}\right]\right\rangle, \\
& S_{p}^{v}=\varphi_{p}\left\langle-\rho_{p}\left[\frac{d v_{p}}{d t}-g_{\eta}\right]\right\rangle, \\
& S_{p}^{w}=\varphi_{p}\left\langle-\rho_{p}\left[\frac{d w_{p}}{d t}-g_{s}\right]\right\rangle,
\end{aligned}
$$

where $\varphi_{p}$ and $\rho_{p}$ are particle mass loading and density, respectively. Apart from the situation where the flow regime is considered as two-way coupling, these terms are neglected. The last term on the right-hand sides of momentum equations reflects the effect of turbulence through the flow field in terms of turbulent stress. In order to solve the RANS equations, extra modelling for Reynolds stress term $\left(\overline{\rho u_{i}^{\prime} u_{j}^{\prime}}\right)$ is required. This term is defined by [30]:

$$
\tau_{i j}=-\rho u_{i}{ }^{\prime} v_{i}{ }^{\prime}=\mu_{t}\left(\frac{\partial U_{i}}{\partial x_{j}}+\frac{\partial U_{j}}{\partial x_{i}}\right)-\frac{2}{3} \rho k \delta_{i j},
$$

where $k$ is the turbulence kinetic energy per unit mass quantified by the mean of the turbulence normal stresses. Thus, it is computed by:

$$
k=\frac{1}{2}\left(\overline{u^{\prime 2}}+\overline{v^{\prime 2}}+\overline{w^{\prime 2}}\right) \text {. }
$$

In the case of this work, among several turbulence models, which are developed to calculate the Reynolds stress term, the two equations of standard $k-\varepsilon$ turbulence model are selected. The turbulence model that can provide excellent performance for flows, in which boundary layers under adverse pressure gradient, separation, and recirculation exist, has been successfully applied to this CFD simulation. The two equations of the standard $k-\varepsilon$ turbulence model to calculate the turbulence kinetic energy, $k$, and dissipation rate, $\varepsilon$, 
are, respectively, written as follows:

$$
\begin{aligned}
& \nabla \cdot(\rho k \vec{V})=\nabla \cdot\left[\frac{\mu_{t}}{\sigma_{k}} \nabla k\right]+2 \mu_{t} S_{i j} \cdot S_{i j}-\rho \varepsilon, \\
& \nabla \cdot(\rho \varepsilon \vec{V})=\nabla \cdot\left[\frac{\mu_{t}}{\sigma_{\varepsilon}} \nabla \varepsilon\right]+C_{1 \varepsilon} \frac{\varepsilon}{k} 2 \mu_{t} S_{i j} . S_{i j}-C_{2 \varepsilon} \rho \frac{\varepsilon^{2}}{k},
\end{aligned}
$$

where $\mu_{t}$ represents the eddy viscosity and is computed as follows:

$$
\mu_{t}=\rho C_{\mu} \frac{k^{2}}{\varepsilon}
$$

In these equations, $S_{i j}$ represents a component of deformation rate, and the model constants are $\sigma_{k}=$ $1.00, \sigma_{\varepsilon}=1.30, C_{1 \varepsilon}=1.44, C_{2 \varepsilon}=1.92$, and $C_{\mu}=0.09$.

\subsection{Particle tracking}

In this step of the study, the sand particles are injected from the pipe inlet into the flow and tracked using the Lagrangian approach. Inspired by Newton's second law, the particle's motion represents a direct consequence of the trade-off between forces acting on them, which is defined by:

$$
m_{p} \frac{d \overrightarrow{V_{p}}}{d t}=\overrightarrow{F_{D}}+\overrightarrow{F_{A}}+\overrightarrow{F_{P}}+\overrightarrow{F_{g}}
$$

where $m_{p}$ is the particle mass, $V_{p}$ is the velocity of the particle, $\overrightarrow{F_{D}}$ is the drag force, $\overrightarrow{F_{A}}$ is the added mass force, $\overrightarrow{F_{P}}$ is the pressure-gradient force, and $\overrightarrow{F_{g}}$ is the resultant of gravity and buoyancy forces. These forces are calculated according to Eqs. (26) to (29) [31]:

$$
\begin{aligned}
& \overrightarrow{F_{D}}=\frac{3 \rho_{f} m_{p} C_{D}}{4 \rho_{p} d_{p}}\left|\overrightarrow{V_{f}}-\overrightarrow{V_{p}}\right|\left(\overrightarrow{V_{f}}-\overrightarrow{V_{p}}\right), \\
& \overrightarrow{F_{A}}=-\frac{1}{2} m_{p} \frac{\rho_{f}}{\rho_{p}} \frac{d \overrightarrow{V_{p}}}{d t} \\
& \overrightarrow{F_{P}}=-\frac{1}{4} \pi d_{p}^{3} \nabla p \\
& \overrightarrow{F_{g}}=m_{p}\left(1-\frac{\rho_{f}}{\rho_{p}}\right) \vec{g} .
\end{aligned}
$$

Herein, $\rho_{f}, V_{f}$, and $d_{p}$ are fluid density, fluid velocity, and particle diameter, respectively. $C_{D}$ is the drag coefficient based on Schiller and Naumann model [32], and:

$$
R e_{p}=\frac{\rho_{f} d_{p}\left|\overrightarrow{V_{f}}-\overrightarrow{V_{p}}\right|}{\mu_{f}},
$$

where $\mu_{f}$ is the dynamic viscosity of fluid; $R e_{p}$ represents the particle Reynolds number. The fourth-order Range-Kutta method is used to solve Eq. (25).
The Crowe model [33] has been used to model the collision of the particle with the wall and the gasliquid interface. The return velocity is usually lower than the collision velocity, and the reason for this drop is the energy transfer from the particle to the wall. The return velocity depends on several important factors: wall and particle material, angle of incidence, and velocity of incidence. According to this model, the particle velocity components after the collision can be calculated as follows

$$
\left\{\begin{array}{l}
v_{x}=v_{x}^{(0)}+\varepsilon_{x} f(e+1) v_{y}^{(0)} \\
v_{y}=-e v_{y}^{(0)} \\
v_{z}=v_{z}^{(0)}+\varepsilon_{z} f(e+1) v_{y}^{(0)}
\end{array}\right.
$$

where $v_{x}^{(0)}, v_{y}^{(0)}, v_{z}^{(0)}$ are the components of the velocity of incidence; $v_{x}, v_{y}, v_{z}$ are the components of return velocity; $f$ represents the skin friction coefficient of wall; $e$ is the coefficient of restitution; and $\varepsilon_{x}$ and $\varepsilon_{z}$ represent the direction cosines on $x-z$ plane. The coefficients of restitution and friction at the wall and interface include $e_{w}=0.95, e_{\text {int }}=0.6, f_{w}=0.3$, and $f_{\text {int }}=0.5$.

\section{Definition of problem and model}

The current research concentrates on a horizontal curved pipe with an internal diameter of $100 \mathrm{~mm}$ and the radius of curvature of $a=300 \mathrm{~mm}$, which carries the two-phase turbulent stratified flow with low liquid holdup. The length of the $90^{\circ}$ curved pipe is $471 \mathrm{~mm}$. Liquid holdup at the bottom of the pipe is assumed to have a depth of $10 \mathrm{~mm}$. In other words, water occupies $5.76 \%$ of the cross-section of pipe; consequently, the bulk of the pipe volume is filled by gas. There is a reasonable assumption during the simulation by which liquid height is considered unchanged. In this situation, the gas phase can be separately modelled, and the effect of liquid phase appears as boundary conditions at the gas-liquid interface. The continuous phase is air with the density of $1.2 \mathrm{~kg} / \mathrm{m}^{3}$ and the dynamic viscosity of $1.51 \times 10^{-5}$ N.s $/ \mathrm{m}^{2}$.

Influenced by centrifugal force, the gas-liquid interface in the bend section is displaced from the horizontal position. Based on the assumption of the smooth interface between gas and liquid, Castillo [2] acquired an analytical expression for angular deviation of gas-liquid interface from the horizontal pipe axis, as depicted in Figure 2 and given as:

$$
\varphi=\frac{w_{l}^{2}}{a g}\left(1-\cos \left(\sqrt{\frac{g \bar{y}}{K_{0}^{2}}} t\right)\right),
$$

where $\varphi$ is the liquid free surface angle with the horizontal surface as shown in Figure 2, $w_{l}$ is the mean stream-wise (axial) velocity of liquid phase, $\bar{y}$ is the distance between cross-sectional center of gravity of liquid 


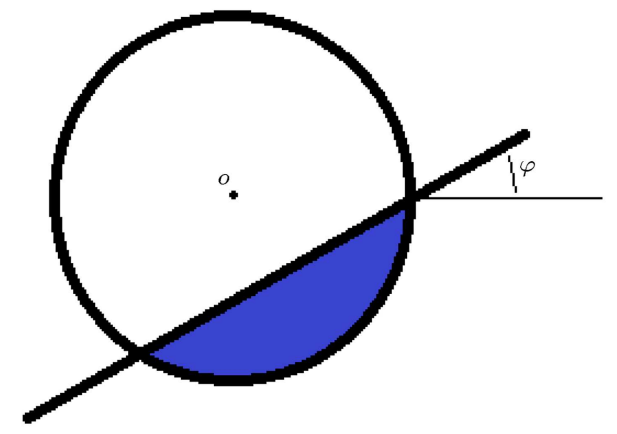

Figure 2. Angular displacement of gas-liquid interface from the horizontal pipe axis.

phase and pipe axis, and $K_{0}$ represents cross-sectional gyration radius of liquid part about the pipe axis. According to Eq. (31), the maximum angular deviation is equal to $2 w_{l}^{2} / \mathrm{ag}$. It is worth mentioning that Eq. (31) is only reasonable for very small angular deviations.

In this investigation, the mean velocities of the liquid and gas phases are considered as $0.02 \mathrm{~m} / \mathrm{s}$ and $4 \mathrm{~m} / \mathrm{s}$, respectively. According to these values and Eq. (31), the maximum displacement is $0.016^{\circ}$. Since the angular deviation is negligible, in this study, the alteration of gas-liquid interface from completely horizontal position has been ignored. In order to ensure that the flow pattern does not vary as the flow passes through the curved section, a qualitative analogy with the experimental results obtained by Vieira et al. [17] has been drawn. Experiments of the gas-liquid stratified wavy flow with gas void fraction of 0.93 , superficial liquid velocity of $0.03 \mathrm{~m} / \mathrm{s}$, and superficial gas velocity of $9 \mathrm{~m} / \mathrm{s}$ passing through the $90^{\circ}$ elbow with curvature ratio, $\delta$, (pipe radius, $R$ /curvature radius, $a$ ) of 0.3342 ; accordingly, the flow pattern was not displaced from the horizontal position. In this work, since the curvature ratio of the considered bend is $0.17(50 / 300)$, the induced centrifugal force is much lower; consequently, the corresponding maximum angular deviation is smaller; therefore, the aforementioned assumption is not far away from the fact.

\section{Numerical method}

The utilization of an extended toroidal coordinate system boosts the development of an effective computational technique to study the stratified flow in curved passages. The computational domain of the gas/liquid flow is illustrated in Figure 3. For the ease of computation, the domain is separated for a given value of liquid holdup; then, the flow fields will be solved independently for each phase by specifying the appropriate boundary conditions at the interfacial surface.

The Navier-Stokes equations, which are expressed in the orthogonal coordinate system $(\xi, \eta, s)$, are used

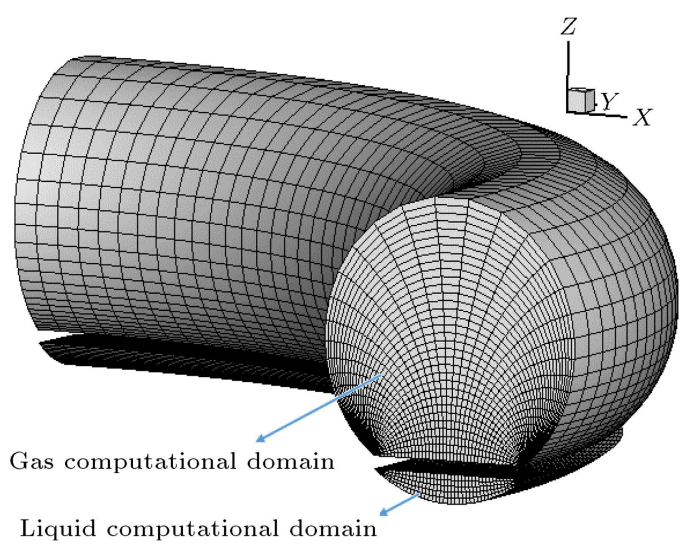

Figure 3. Computational domain of the gas and liquid flows.

to describe the gas phase flow field through the bend. A finite volume method on staggered grids is used to discretize the governing equations. Central difference and QUICK scheme are used to discretize the diffusion and convection terms, respectively. Moreover, the $k-\varepsilon$ model is employed to reflect the nature of turbulence in the flow field.

In terms of boundary conditions, at the walls, the impenetrability and no-slip boundary condition are enforced. At the inlet, a Poiseuille velocity distribution has been considered, while a constant pressure outflow at the outlet of the curved pipe is applied. The main purpose of this work is to predict the gas phase velocity field; therefore, a simplified assumption at the gas/liquid interface has been made based on which the axial velocity of interface is constant during the simulation and equal to the mean axial velocity of the liquid phase, and the other components of the velocity are considered zero.

In order to validate the present CFD code, the simulation results have been compared with the experimental data by Bovendeerd et al. [34] to develop singlephase flow in a $90^{\circ}$ bend pipe. Herein, pipe internal radius is $4.0 \mathrm{~mm}$, radius of curvature is $24.0 \mathrm{~mm}$, and Reynolds number is 700. In Figures 4 and 5, the predicted dimensionless axial velocity profile on the symmetry plane in two sections of the curve at $z=1$ and $1.7\left(z=R \theta /(a R)^{0.5}\right.$, where $R$ and $\theta$ are pipe radius and axial direction along the curve, respectively, as shown in Figure 6) is compared with the experimental measurements by Bovendeerd et al. [34]. The vertical axis in these figures represents the dimensionless axial velocity, which is made non-dimensional with the mean axial velocity. On the other hand, the horizontal axis is dimensionless distance from the outer wall of the bend ( $x_{1}$ is the distance from the outer wall). It can be seen that the predicted dimensionless axial velocity profiles are in good agreement with the experimental measurements.

To examine the validation of the present model 


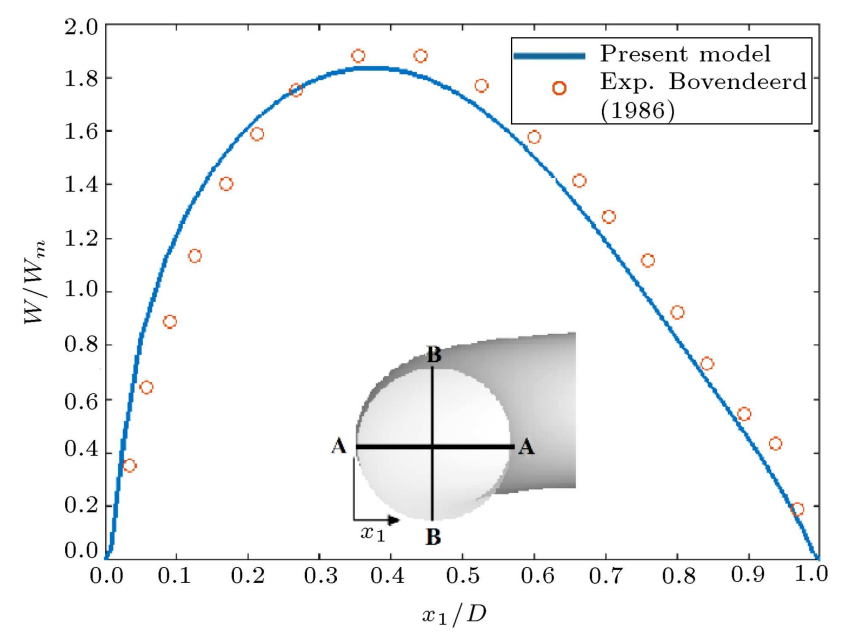

Figure 4. The dimensionless axial velocity profile on the AA plane; $z=1, \delta=1 / 6, R e=700$ [34].

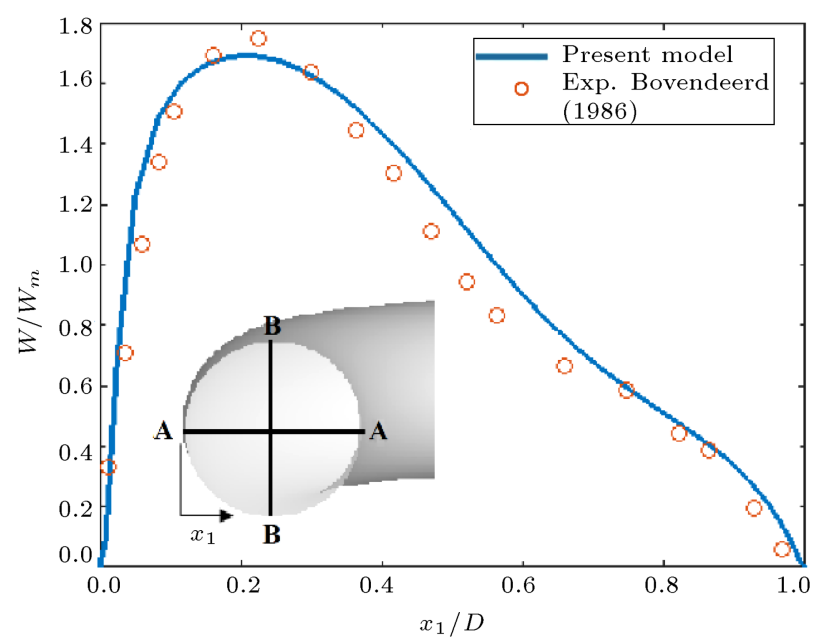

Figure 5. The dimensionless axial velocity profile on the AA plane; $z=1.7, \delta=1 / 6, R e=700$ [34].

for the stratified gas-liquid flows, a condition in which the bend radius of curvature tends towards infinity is considered, and the results are compared with those for the straight pipes. The predicted dimensionless axial velocity profile of the gas-liquid phase for fully developed stratified flow in a straight pipe is compared with the analytical solution provided by Brauner et al. [35] in Figure 7. The axial velocities have been made non-dimensional by:

$$
V_{R}=\frac{R^{2}}{4 \mu_{G}} \frac{d p}{d s} .
$$

For this case, to obtain the exact value of the interfacial velocity, its value has been initialized; then, the gas phase equations are solved, and axial gas velocity profile is evaluated. In the next step, the corresponding averaged shear stress at the interface is calculated and, consequently, is applied to the liquid phase.

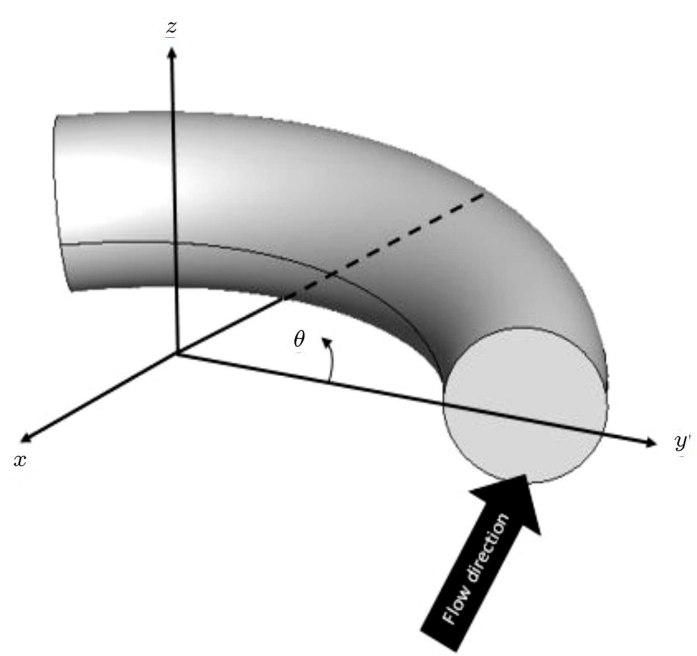

Figure 6. Computational domain of the bend.

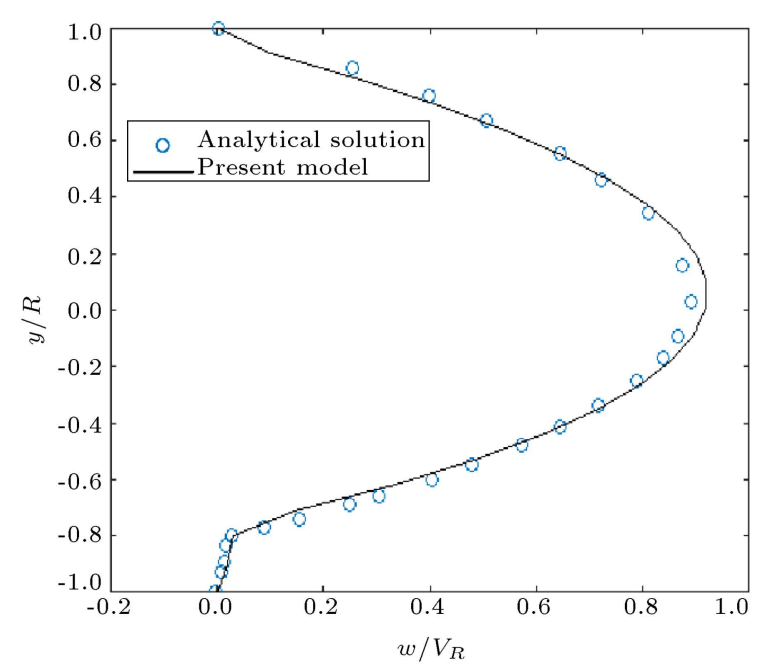

Figure 7. Comparison of the predicted dimensionless axial velocity profile along the vertical centerline with the analytical solution of Brauner et al. [35]; $h_{L}=D / 10$, $\mu_{G} / \mu_{L}=0.01$.

Eventually, the liquid phase is solved, and the interface velocity is calculated. The new interface velocity is then applied to the gas phase, and the solution process will be repeated until the gas-liquid interfacial velocity becomes unchanged and the solution converges. Figure 7 demonstrates that the present model prediction is in excellent agreement with the analytical solution.

Lastly, to validate turbulence in the fully developed pipe flow, the results are compared with experimental ones provided by Laufer [36]. In experiments, pipe internal radius is $127 \mathrm{~mm}$, and the radius of curvature tends towards infinity (straight pipe) carrying flow with Reynolds number of 50000 . This comprehensive comparison consists of three items including axial velocity profile, turbulence kinetic energy, and dissipation rate displayed in Figures 8, 9, and 10, respectively. These parameters have been made dimensionless by: 


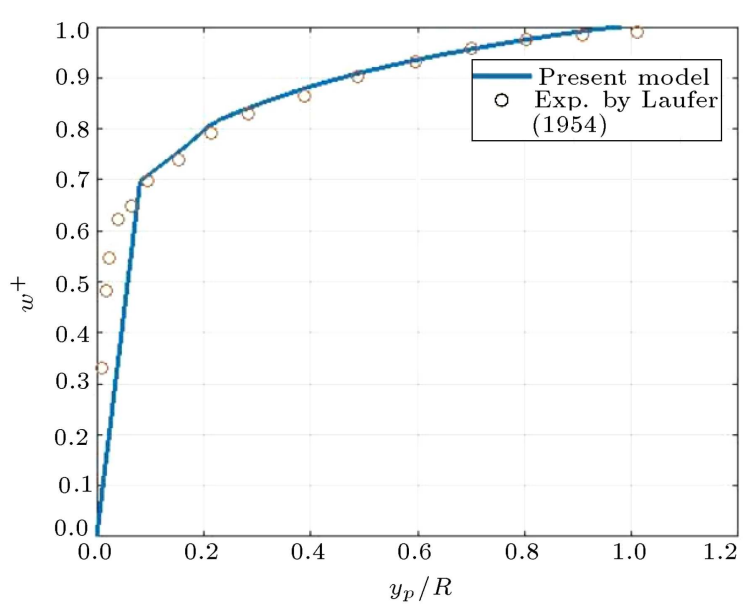

Figure 8. Comparison of the predicted dimensionless axial velocity profile along the vertical centerline with the experimental results by Laufer [36].

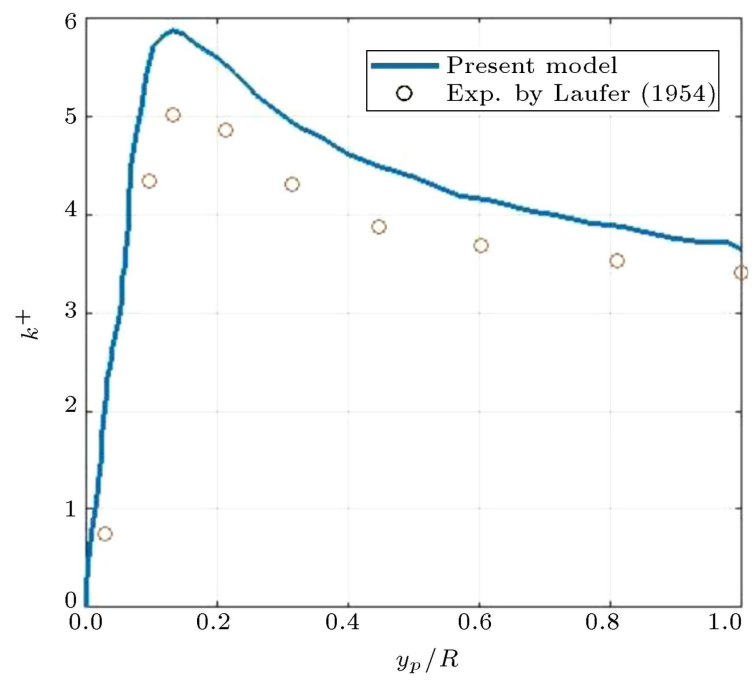

Figure 9. Comparison of the predicted dimensionless turbulence kinetic energy profile along the vertical centerline with the experimental results by Laufer [36].

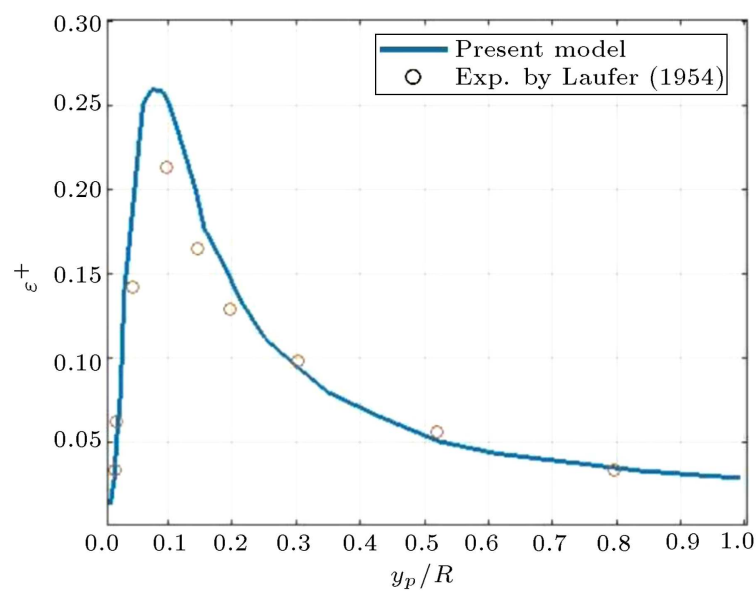

Figure 10. Comparison of the predicted dimensionless turbulence dissipation rate profile along the vertical centerline with the experimental results by Laufer [36].

$$
\begin{aligned}
w^{+} & =\frac{w}{w_{\max }}, \\
k^{+} & =\frac{k}{w_{\tau}^{2}}, \\
\varepsilon^{+} & =\frac{\mu \varepsilon}{\rho w_{\tau}^{4}} .
\end{aligned}
$$

Although comparing the CFD simulation results with axial velocity and turbulence parameters measured experimentally revealed a satisfactory agreement (discrepancy $< \pm 15 \%$ ), there is a slight deviation near the wall mainly caused by the difference between initial $y^{+}$and actual one leading to the right selection of appropriate turbulence model.

\section{Results and discussion}

When the two-phase flow passes through the bend, the maximum axial velocity of the gas phase tends towards the outer wall, and the secondary flow forms and evolves along the pipe. Figure 11 shows axial velocity contours, and Figure 12 displays the corresponding secondary flows occurring in the gas phase through the curved pipe in four axial sections: $s=0.03 \mathrm{~m}, 0.1 \mathrm{~m}$, $0.14 \mathrm{~m}$, and $0.18 \mathrm{~m}$. The gas Reynolds number is about 27000 in all cases.

Figure 11 depicts that the central maximum stream-wise velocity region disappears gradually and shifts to the region farther away from the inner wall (left) and closer to the gas-liquid interface, top and outer pipe wall (right). Near the inner wall, the axial velocity magnitude is very low, while it is quite high near the outer wall. Therefore, the radial velocity gradient is very high at the outer wall, although this gradient is much lower between the inner wall and maximum axial velocity region. The contour lines of the axial velocity are asymmetric with respect to horizontal centerline, and this asymmetric behavior becomes noticeable along the pipe from $s=0.03 \mathrm{~m}$ to $s=0.18 \mathrm{~m}$. At $s=0.18 \mathrm{~m}$, the contours seem to be highly skewed.

Figure 12 shows that, at $s=0.03 \mathrm{~m}$, the secondary flows in the gas phase generate four vortex zones. Two vortices are in the vicinity of gas-liquid interface and pipe wall, while the other two larger vortices are created in the upper part of the pipe crosssection. By drawing an analogy among the secondary flows in all axial sections, as provided in Figure 12, it can be understood that the two lower vortices located in the corners of gas-liquid interface and that near the inner wall become stronger along the pipe, while the other one does not vary significantly.

In this step, the behaviour of the entrained particles through the pipe carrying considered flow will be examined. Table 1 contains the parameters describing the geometry, flow, and entrained particles. 

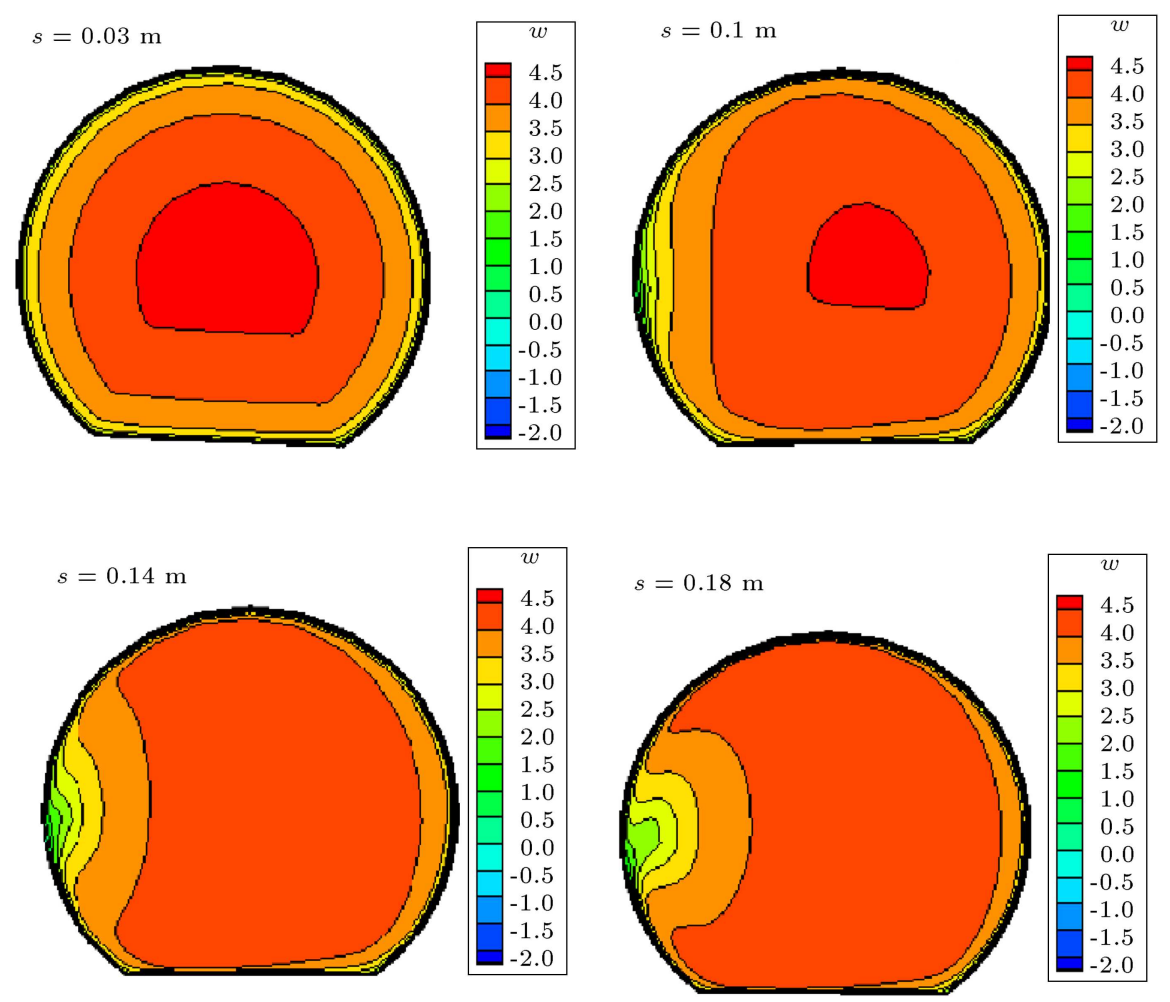

Figure 11. Axial velocity contours in the gas phase at $s=0.03,0.1,0.14$, and 0.18 .
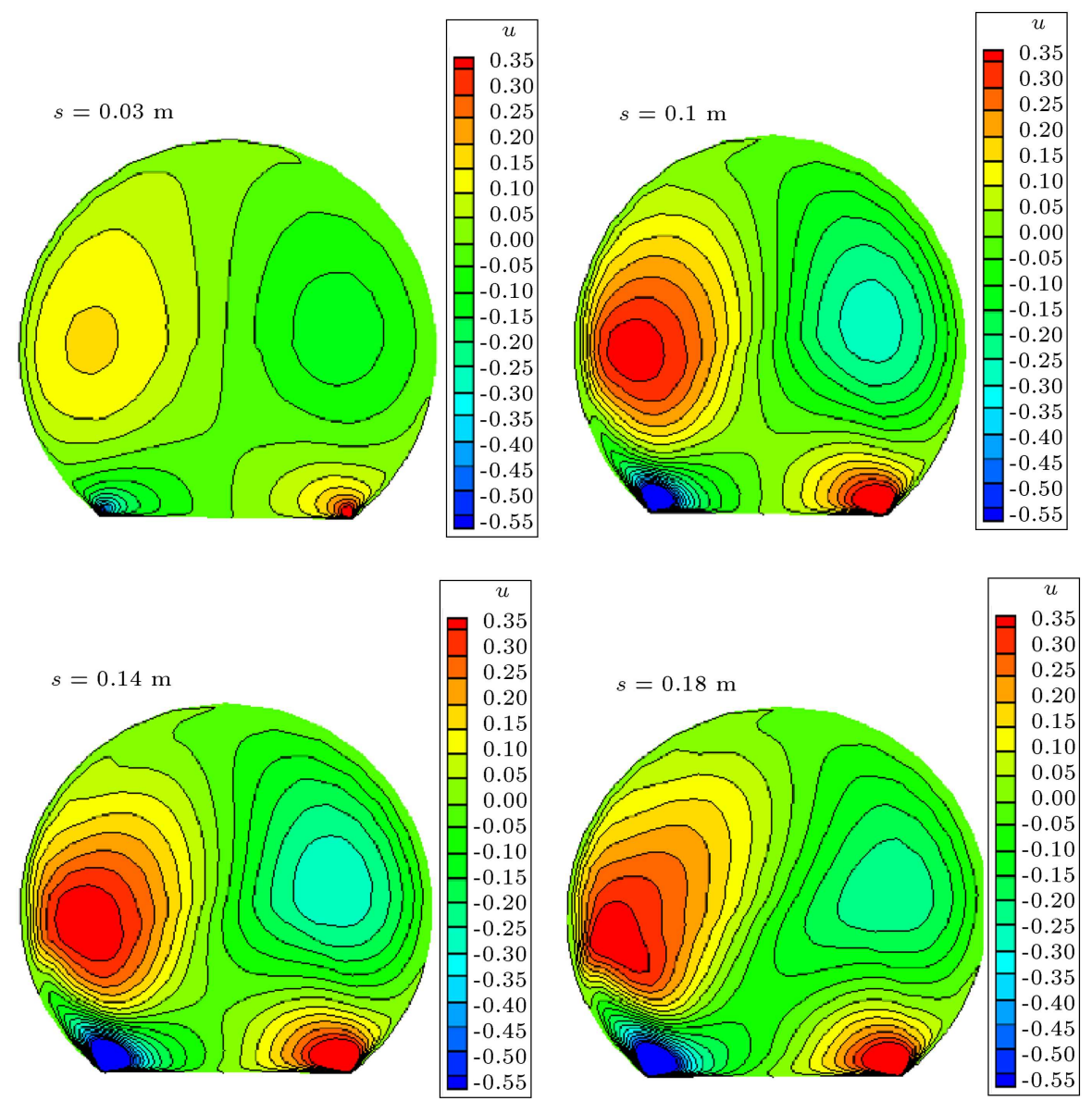

Figure 12. Secondary flow in the gas phase at $s=0.03,0.1,0.14$, and 0.18 . 
Table 1. Simulated cases in particle tracking section.

\begin{tabular}{lc}
\hline Pipe orientation & $H-H$ \\
Bend length & $0.2 \mathrm{~m}$ \\
Curvature radius & $0.1274,0.25,0.3 \mathrm{~m}$ \\
Particle diameter & $200 \mu \mathrm{m}$ \\
Particle density & $2500 \mathrm{~kg} / \mathrm{m}^{3}$ \\
Particle initial velocity & $70 \%$ of gas velocity \\
Gas velocity & $2 \mathrm{~m} / \mathrm{s}$ \\
Interface velocity & $0.01 \mathrm{~m} / \mathrm{s}$ \\
Liquid film thickness & $0.01 \mathrm{~m}$ \\
\hline
\end{tabular}

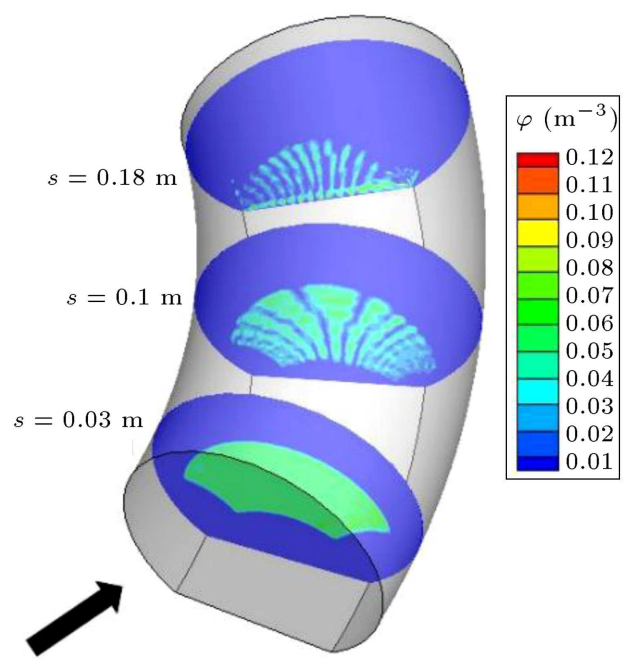

Figure 13. Concentration of particles in the bend (curvature radius $=0.3 \mathrm{~m}$ ).

The particle injection region from the inlet surface is ranged as in the following fashion:

$$
\left\{\begin{array}{l}
0.8511 \leq \xi \leq 1.8998 \\
-0.8306 \leq \eta \leq 0.8306
\end{array}\right.
$$

According to Figure 13, the particles are forced to move towards the outer wall during the passage through the bend influenced by the centrifugal force. Moreover, they are continuously under the influence of gravitational force. On the other hand, pressure gradient in the radial direction occurs in the flow field; therefore, in each section of the bend, the trade-off between the aforesaid forces is different, which plays a key role in particles' motion. Near the internal walls, the dominant force is the gravity, and the particles tend to have lower concentration than those near the outer wall of the bend.

As shown in Figures 14 and 15, by decreasing the radius of curvature, the particles are more oriented towards the inner wall. The particles are mostly inclined to the vortex located on the right side of the pipe, near the interface, and are still more likely to collide with the interface.

Furthermore, in order to investigate the effect of

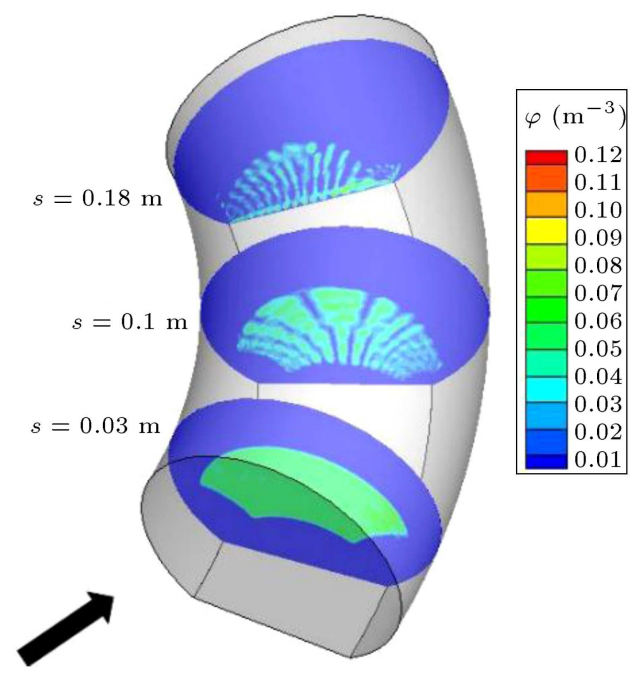

Figure 14. Concentration of particles in the bend (curvature radius $=0.25 \mathrm{~m}$ ).

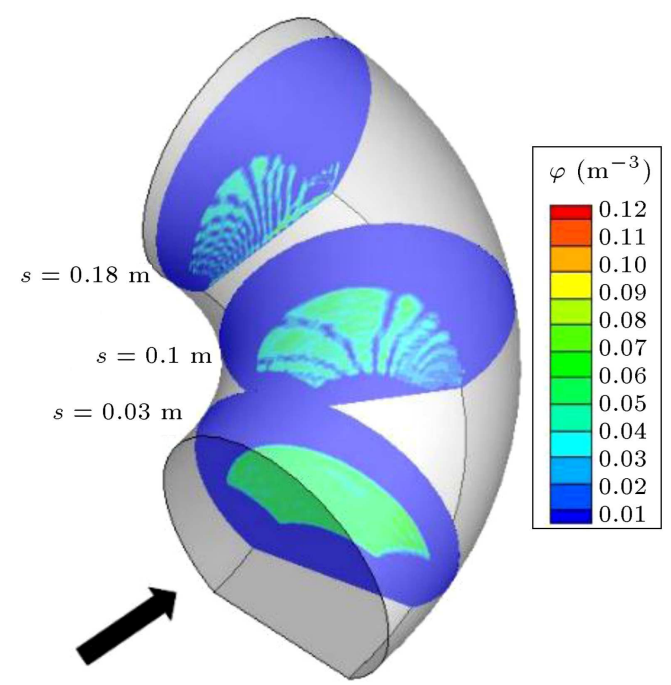

Figure 15. Concentration of particles in the bend (curvature radius $=0.1274 \mathrm{~m}$ ).

particle diameter on their path, three particle diameters of 5, 50, and 200 microns are injected from the center of inlet surface. The direction of movement of these particles is depicted in Figure 16. By decreasing the diameter of the particles, the pressure-gradient force, the added mass force, and the gravity force decrease with the third power of the diameter; however, the drag force decreases with the first power of the particle diameter; consequently, centrifugal force acting on the particles overcomes other forces and pushes the particles towards the outer wall.

\section{Conclusions}

To simulate the gas-liquid stratified flow in a horizontal curved pipe, a new toroidal coordinate system was developed and utilized for convenient mapping of the 


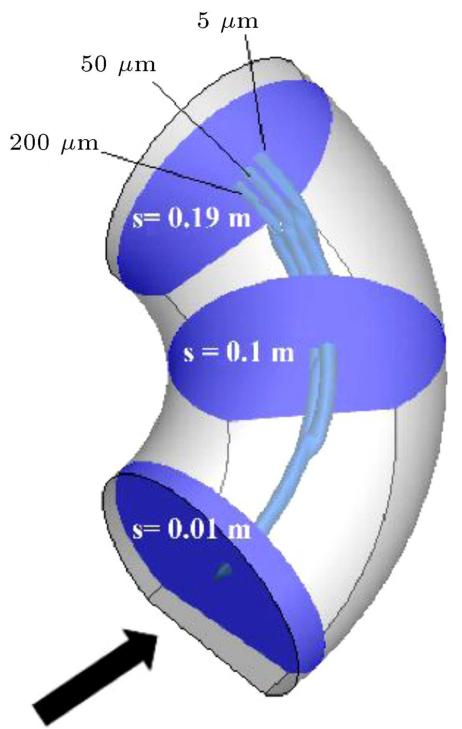

Figure 16. Comparison of motion of particles with different sizes.

physical domain and the gas-liquid interface. The 3-D steady-state momentum equations were discretized and solved using the finite volume scheme. By comparing the numerical data with the previous experimental results, the validity of the numerical model was examined, showing that the new model could precisely handle developed stratified gas-liquid flows in curved pipe, particularly at low radius of curvature.

In general, higher axial velocities are observed in the gas phase. Based on the horizontal gas-liquid flow simulation, the maximum velocity was detected close to halfway between the interface and the upper wall. This could be attributed to the secondary flows similar to the results acquired by Meknassi et al. [37]. The current results confirm the presence of secondary flows in the gas phase, indicating that the flow is not fully developed.

The present results illustrate that the contour lines of the axial velocity in gas-liquid stratified flow are asymmetric with respect to horizontal centerline. In the axial section from $s=0.03 \mathrm{~m}$ to $0.18 \mathrm{~m}$, the secondary flow formed four major vortex (recirculation) zones. Furthermore, the two secondary vortices near the corners of the gas-liquid interface are found to be stronger than the other vortices are and exist in all sections in the curved pipe.

Entrained particle tracking was performed by means of the Lagrangian approach considering the twoway coupling regime between the flow and particles. To solve the equation of particle's motion, the fourthorder Range-Kutta method was exploited. Finally, the results obtained by the particle tracking indicate that, in each section of the bend, the trade-off between centrifugal and pressure gradient forces plays a key role in particles' motion close to the inner surface; in addition, the dominant force is the gravity, and the particles tend to have lower concentration than those close to the outer wall of the bend. In terms of the effect of particle size, decreasing the particle's diameter results in enhancing the centrifugal force, which pushes the particles towards the outer wall. Furthermore, the comparison of particles' motion through bends with various curvature demonstrates that by decreasing the radius of curvature, the particles are more oriented towards the inner wall. The particles are mostly inclined to the vortex located on the right side of the pipe, near the interface, and are still more likely to collide with the interface.

\section{Nomenclature}

$\begin{array}{ll}a & \text { Curvature radius }(\mathrm{m}) \\ D & \text { Pipe diameter }(\mathrm{m}) \\ c & \text { Focal length in bipolar coordinate } \\ & \text { system }(\mathrm{m}) \\ h_{L} & \text { Liquid height }(\mathrm{m}) \\ h_{\eta}, h_{\xi}, h_{s} & \text { Scale factors } \\ R & \text { Pipe radius }(\mathrm{m}) \\ p & \text { Pressure }(\mathrm{Pa}) \\ s & \text { Axial direction }(\mathrm{m}) \\ u & \text { Velocity component in } \eta \text { direction } \\ & \text { (ms }{ }^{-1} \text { ) } \\ v & \text { Velocity component in } \xi \text { direction } \\ & \text { (ms }{ }^{-1} \text { ) } \\ w & \text { Axial velocity }\left(\mathrm{ms}^{-1}\right) \\ w_{\text {mean }} & \text { Axial velocity }\left(\mathrm{ms}^{-1}\right) \\ X, Y, Z & \text { Cartesian coordinates }(\mathrm{m}) \\ k & \text { Turbulence kinetic energy } \\ g & \text { Gravity }\end{array}$

\section{Greek letters}

Half-angle subtended by liquid at the center of pipe

$\delta \quad$ Curvature ratio

$\eta, \xi \quad$ Bipolar coordinates

$\theta \quad$ Stream-wise angle

$\mu \quad$ Viscosity (Pa.s)

$\rho \quad$ Density $\left(\mathrm{kg} . \mathrm{m}^{-3}\right)$

$\varphi \quad$ Angular displacement of gas-liquid interface about the pipe axis (radian)

$\varepsilon \quad$ Turbulence dissipation rate

\section{Subscripts}

$\begin{array}{ll}G & \text { Gas } \\ L & \text { Liquid }\end{array}$


$\eta, \xi, s \quad$ New toroidal coordinates

$p$ Particle

\section{References}

1. Cohen, M.I. "An investigation of pressure drop in a two-phase two-component flow in bends", M.Sc. Thesis, MIT (1957).

2. Castillo, J.R. "Study of two-phase flow in pipe bends", M.Sc. Thesis, MIT (1957).

3. Grander, G.C. and Neller, P.H. "Phase distributions in flow of an air-water mixture round bends and past obstructions at the wall of a $76-\mathrm{mm}$ bore tube", Institution of Mechanical Engineers, 184, pp. 93-101 (1969).

4. Usui, K., Aoki, S., and Inoue, A. "Flow behavior and pressure drop of two-phase flow through C-shaped bend in vertical plane (I)", Journal of Nuclear Science and Technology, 17(12), pp. 875-887 (1980).

5. Usui, K., Aoki, S., and Inoue, A. "Flow behavior and phase distribution in two-phase flow around inverted U-bend", Journal of Nuclear Science and Technology, 20(11), pp. 915-928 (1983).

6. Das, S.K., Biswas, M.N., and Mitra, A.K. "NonNewtonian liquid flow in bends", The Chemical Engineering Journal, 45(3), pp. 165-171 (1991).

7. Bandyopadhyay, T.K., Banerjee, T.K., and Das, S.K. "Gas-non-newtonian liquid flow through elbows", Chemical Engineering Communications, 182(1), pp. 21-33 (2000).

8. Ribeiro, A.M., Bott, T.R., and Jepson, D.M. "The influence of a bend on drop sizes in horizontal annular two-phase flow", International Journal of Multiphase Flow, 27(4), pp. 721-728 (2001).

9. Azzi, A., Friedel, L., Kibboua, R., and Shannak, B. "Reproductive accuracy of two-phase flow pressure loss correlations for vertical $90^{\circ}$ bends", Forschung im Ingenieurwesen, 67(3), pp. 109-116 (2002).

10. Wang, C.-C., Chen, I.Y., Yang, Y.W., and Chang, Y.J. "Two-phase flow pattern in small diameter tubes with the presence of horizontal return bend", International Journal of Heat and Mass Transfer, 46(16), pp. 2975-2981 (2003).

11. Wang, C.-C., Youn Chen, I., Yang, Y.W., and Hu, R. "Influence of horizontal return bend on the two-phase flow pattern in small diameter tubes", Experimental Thermal and Fluid Science, 28(2-3), pp. 145-152 (2004).

12. Wang, C.-C., Chen, I.Y., and Huang, P.-S. "Two-phase slug flow across small diameter tubes with the presence of vertical return bend", International Journal of Heat and Mass Transfer, 48(11), pp. 2342-2346 (2005).

13. Wang, C.-C., Chen, I.Y., Lin, Y.-T., and Chang, Y.J. "A visual observation of the air-water two-phase flow in small diameter tubes subject to the influence of vertical return bends", Chemical Engineering Research and Design, 86(11), pp. 1223-1235 (2008).
14. Spedding, P.L., Benard, E., and McNally, G.M. "Twoand three-phase flow through a 90 degree bend", Developments in Chemical Engineering and Mineral Processing, 13(5-6), pp. 719-730 (2005).

15. Abdulkadir, M., Zhao, D., Sharaf, S., Abdulkareem, L., Lowndes, I.S., and Azzopardi, B.J. "Interrogating the effect of $90^{\circ}$ bends on air-silicone oil flows using advanced instrumentation", Chemical Engineering Science, 66(11), pp. 2453-2467 (2011).

16. Kesana, N. "Erosion in multiphase pseudo-slug flow with emphasis on sand sampling and pseudo-slug characteristics", The University of Tulsa, Oklahoma, USA (2013).

17. Vieira, R.E., Kesana, N.R., McLaury, B.S., Shirazi, S.A., Torres, C.F., Schleicher, E., and Hampel, U. "Experimental investigation of the effect of $90^{\circ}$ standard elbow on horizontal gas-liquid stratified and annular flow characteristics using dual wire-mesh sensors", Experimental Thermal and Fluid Science, 59, pp. 7287 (2014).

18. Mokhtarifar, N., Saffaraval, F., Saffar-Avval, M., Mansoori, Z., and Siamie, A. "Experimental modeling of gas-solid heat transfer in a pipe with various inclination angles", Heat Transfer Engineering, 36(1), pp. 113-22 (2015).

19. Nojabaei, B., Mansoori, Z., and Saffar-Avval, M. "Heat transfer in turbulent liquid-solid flow considering the interparticle collision effect", Petroleum Science and Technology, 13;30(12), pp. 1296-306 (2012).

20. Pishvar, M., Avval, M.S., Mansoori, Z., and Amirkhosravi, M. "Three dimensional heat transfer modeling of gas-solid flow in a pipe under various inclination angles", Powder Technology, 1(262), pp. 223-32 (2014).

21. Dabirian, R., Mansouri, A., Mohan, R., Shoham, O., and Kouba, G. "CFD simulation of turbulent flow structure in stratified gas/liquid flow and validation with experimental data", In SPE Annual Technical Conference and Exhibition (2015).

22. Haj, A., Mansoori, Z., Saffar Avval, M., and Ahmadi, G. "Developing stratified gas-liquid flow 3-D numerical simulation in curved pipes", 9th International Conference on Multiphase Flow, Firenze, Italy (2016).

23. Ayati, A.A., Kolaas, J., Jensen, A., and Johnson, G.W. "PIV in stratified gas-liquid flow in a horizontal pipe using water droplets as tracers in the gas-phase", arXiv preprint arXiv:1701.00387 (2017).

24. Vest $\phi 1$, S., Kumara, W.A., and Melaaen, M.C. "Particle image velocimetry measurements of stratified gasliquid flow in horizontal and inclined pipes", Multiphase Flow: Theory and Applications, 417 (2018).

25. Stevenson, P. and Thorpe, R.B. "Velocity of isolated particles along a pipe in stratified gas-liquid flow", AIChE Journal, 48(5), pp. 963-9 (1 May 2002).

26. Dabirian, R., Mohan, R.S., Shoham, O., and Kouba, G. "Solid-particles flow regimes in air/water stratified flow in a horizontal pipeline", Oil and Gas Facilities, 15(06) (2016). 
27. Zehtabiyan-Rezaie, N., Damirchi-Darasi, S.R., Zarandib, M.F., and Saffar-Avval, M. "Generalized heat transfer and entropy generation of stratified air-water flow in entrance of a mini-channel", Scientia Iranica, Transactions B, Mechanical Engineering, 24(5), pp. 2406-17 (2017).

28. Banakermani, M.R., Naderan, H., and Saffar-Avval, M. "An investigation of erosion prediction for $15^{\circ}$ to $90^{\circ}$ elbows by numerical simulation of gas-solid flow", Powder Technology, 334, pp. 9-26 (2018).

29. Hughes, W.F., Gaylord, E.W., and Hughes, G.W. Basic Equations of Engineering Science, New York: McGraw-Hill (1964).

30. Versteeg, H.K. and Malalasekera, W. "An introduction to computational fluid dynamics: The finite volume method", Longman Scientific and Technical (1995).

31. Wang, K., Li, X., Wang, Y., and He, R. "Numerical investigation of the erosion behavior in elbows of petroleum pipelines", Powder Technology, 314, pp. 490-9 (2017).

32. Naumann, Z. and Schiller, L. "A drag coefficient correlation", Z. Ver Deutsch. Ing., 77, pp. 318-23 (1933).

33. Crowe, C.T., Schwarzkopf, J.D., Sommerfeld, M., and Tsuji, Y., Multiphase Flows with Droplets and Particles, CRC press (2011).

34. Bovendeerd, P.H.M., Steenhoven, A.A.V., Vosse, F.N.V.D., and Vossers, G. "Steady entry flow in a curved pipe", Journal of Fluid Mechanics, 177, pp. 233-246 (1987).

35. Brauner, N., Rovinsky, J., and Maron, D.M. "Analytical solution for laminar-laminar two-phase stratified flow in circular conduits", Chemical Engineering Communications, 141-142(1), pp. 103-143 (1996).

36. Laufer, J. "The structure of turbulence in fully developed pipe flow", NACA Rep. no. 1174 (1954).

37. Meknassi, F., Benkirane, R., Liné, A., and Masbernat, L. "Numerical modeling of wavy stratified two-phase flow in pipes", Chemical Engineering Science, 55(20), pp. 4681-97 (2000).

\section{Biographies}

Mohammad Ali Rasoulian received his BSc degree in Mechanical Engineering from Ferdowsi University of Mashhad, Mashhad, Iran in 2011 and his MSc degree in Mechanical Engineering from Amirkabir University of Technology (Tehran Polytechnic), Tehran, Iran in 2014. Currently, He is a Research Assistant at Energy Research Center of Amirkabir University of Technology. His research interests include computational fluid dynamics (CFD), particle transport, windbreaks and wind flows, solid particle erosion, and multiphase flows.

Amin Rasteh received his BSc degree in Mechanical Engineering from Yasuj University, Iran in 2011 and his
MSc degree in Mechanical Engineering from Amirkabir University of Technology (Tehran Polytechnic), Iran in 2014. He is currently a Research Assistant at Energy Research Center in Amirkabir University of Technology. His research interests include computational fluid dynamics, convection heat transfer, solid particle erosion, multiphase flows, and turbomachines.

Ali Farokhipour received his BS degree in Aerospace Engineering from K. N. Toosi University of Technology, Iran in 2011 and his MS degree in Mechanical Engineering from Amirkabir University of Technology (Tehran Polytechnic), Iran in 2013, where he is currently a $\mathrm{PhD}$ degree student. His research interests include computational fluid dynamics, gas turbine, non-premixed combustion, solid particle erosion, and multiphase flows.

Zohreh Mansoori completed her PhD in Mechanical Engineering from Amirkabir University of Technology (Polytechnic), Tehran, Iran. She is an Associate Professor and the Head of Energy Research Center in Amirkabir University of Technology. She has published more than 40 papers in reputable journals and has been serving as an Editorial Board Member of repute.

Majid Saffar-Avval is a Professor of Mechanical Engineering at Amirkabir University of Technology (AUT), Tehran, Iran. He received his BSc and MSc degrees from Sharif University of Technology and his PhD degree from the Ecole Nationale des Arts et Metiers (ENSAM), Paris, France in 1985. He has been teaching at AUT since then. He was the Head of the Mechanical Engineering Department from June 2000 to June 2002 and the Head of the Energy and Control Center of Excellence from May 2007 to March 2012 at AUT. His research contributions are in the fields of twophase heat transfer, advanced thermal systems, energy management, and bio-heat transfer.

Ali Haj received his MSc degree in Mechanical Engineering from Amirkabir University of Technology (Tehran Polytechnic), Iran in 2016. His research interests include heat transfer enhancement, two-phase flow modelling, and Computational Fluid Dynamics (CFD).

Goodarz Ahmadi received his PhD degree in Mechanical Engineering from the Purdue University, West Lafayette, IN, USA, and is now a Professor of Mechanical Engineering at Clarkson University, USA. His research work concerns particle transport, deposition and removal, aerosol mechanics, stochastic processes in engineering, advanced theory of turbulence, and multiphase flow modelling. 